Meta

Journal des traducteurs

Translators' Journal

\title{
La traduction juridique : standardisation versus créativité
}

\section{Vassilis G. Koutsivitis}

Volume 35, numéro 1, mars 1990

Actes du colloque international " La traduction proligère "

URI : https://id.erudit.org/iderudit/003346ar

DOI : https://doi.org/10.7202/003346ar

Aller au sommaire du numéro

Éditeur(s)

Les Presses de l'Université de Montréal

ISSN

0026-0452 (imprimé)

1492-1421 (numérique)

Découvrir la revue

Citer cet article

Koutsivitis, V. G. (1990). La traduction juridique : standardisation versus créativité. Meta, 35(1), 226-229. https://doi.org/10.7202/003346ar d'utilisation que vous pouvez consulter en ligne.

https://apropos.erudit.org/fr/usagers/politique-dutilisation/ 


\section{LA TRADUCTION JURIDIQUE : STANDARDISATION VERSUS CRÉATIVITÉ}

VASSILIS G. KOUTSIVITIS

Commission des Communautés européennes, Bruxelles, Belgique

La traduction juridique couvre plusieurs domaines en plein essor actuellement: législation nationale et internationale (États plurilingues, organisations internationales, traités entre États), textes judiciaires, contrats privés, ouvrages de doctrine, etc. On considère généralement qu'elle appartient à une catégorie de traductions plus large, la traduction scientifique et technique ou traduction spécialisée, tout en gardant ses propres particularités. Les controverses commencent quand il s'agit de délimiter l'étendue et la teneur de ces particularités. Signalons-en deux parmi les plus importantes: la traduction juridique est une traduction technique mais aussi culturelle; elle est scientifique mais aussi sociale (Weston, 1988: 681-682). Sa technicité consiste en ses outils spécialisés, son caractère culturel repose sur le fait qu'elle se réfère constamment à des institutions humaines différentes d'un pays à l'autre. Son aspect scientifique se concrétise par sa méthode rigoureuse, sa dimension sociale se reflète dans son adaptation continue et son évolution dynamique.

En raison de son caractère complexe, il est normal que certains insistent sur l'un de ses aspects et que d'autres accentuent une autre de ses facettes. En généralisant (dangereusement), on pourrait dire que les juristes (je parle de ceux qui ne sont pas des traducteurs professionnels) préféreraient, le cas échéant, sacrifier le génie de la langue-cible ou violer telle ou telle méthode traductionnelle pour sauvegarder la technicité d'un texte juridique. À l'inverse, les traducteurs qui proviennent d'autres horizons succombent parfois à la tentation d'adopter une attitude trop libérale (je dirais libertaire) à l'égard d'un texte juridique qui exige une prudence accrue.

Une caractéristique importante de la traduction juridique est son caractère contraignant qui lui impose une série de... contraintes. Ici aussi, il faut relativiser les choses.

Il est évident qu'une traduction juridique peut avoir des conséquences importantes pour le justiciable. Mais n'en est-il pas de même avec un manuel technique pour le consommateur, un texte médical pour le patient ou une étude financière pour les acteurs économiques? L'ordre de grandeurs peut être différent, mais le problème de fond reste le même.

Le traducteur juridique est donc constamment sous la pression de forces opposées: d'un côté, rester le plus près possible du texte d'origine, respecter les précédents, pour éviter toute imprécision, tout malentendu, pour ne pas donner lieu à des interprétations tendancieuses et, de l'autre côté, axer son effort sur la compréhension du message et le reformuler librement dans la langue de traduction. La dichotomie qui caractérise le texte juridique, entre le langage normalisé d'un côté et le discours libre de l'autre, devient la contradiction inhérente au travail du traducteur juridique; celui-ci est appelé à la dépasser, à lui donner une issue positive. Suivre la norme ou créer?

Pour apporter une réponse réfléchie et utile à cette question, essayons d'abord de voir plus concrètement le cadre et ses composantes. 
Répétons que la traduction juridique est une traduction technique et culturelle, scientifique et sociale. Elle est variée (législative, judiciaire, administrative, commerciale, théorique, etc.) et souvent imprégnée du style personnel de l'auteur et de sa position dans la hiérarchie des émetteurs. Elle demande donc un effort accru de la part du traducteur dans les domaines de la documentation et de la terminologie, d'une part, mais également de la stylistique et de la rédaction. Elle peut ainsi provoquer deux attitudes fondamentales de la part du traducteur: une tendance à la transposition lexicale, littérale ou une approche interprétative visant à l'équivalence fonctionnelle.

La fidélité aux précédents, le rôle important des citations, la terminologie technique, les formules stéréotypées sont autant de facteurs qui poussent le traducteur vers la première attitude. En ce qui concerne les termes, élément aussi capital que commun dans toute traduction technique, ils doivent être parfaitement parallèles en langue-source et langue-cible. Le terme traduit doit, «au-delà d'un sens fondamental, (avoir) la totalité des qualités terminologiques» (Ferland, 1987: 14) du terme original. Quant aux formules standardisées, il s'agit d'énoncés répétitifs qui se trouvent surtout dans les parties introductive et finale des textes juridiques et qui, en raison du caractère protocolaire et solennel de ces textes, deviennent rigides et finalement figés, ne permettant pas au traducteur d'y échapper d'aucune manière.

Mais cela est seulement un côté de la médaille. La traduction juridique implique un effort aussi important, sinon plus, d'interprétation et de création. Les textes juridiques sont le produit d'auteurs pour qui l'expression linguistique est l'outil primordial. Il n'en est pas de même pour les textes des sciences exactes, où le support linguistique est secondaire. Pour l'auteur juridique, chaque mot est pensé et repensé, chaque phrase est formulée et reformulée. Le message est complexe, l'intention souvent cachée.

Le deuxième rédacteur, qui est le traducteur, ne peut pas par conséquent se contenter, par une vitesse acquise, de transcoder la totalité du texte juridique. Il doit identifier sa partie libre, rechercher le vouloir-dire de l'auteur, l'interpréter et le reformuler dans la langue-cible. Les textes juridiques sont peut-être les seuls textes qui invitent à l'interprétation - je dirais qu'ils la provoquent, d'une manière explicite quasi officielle. La méthode de l'interprétation est un des piliers de la construction juridique. L'interprétation est une institution juridique. Tout texte juridique est potentiellement l'objet de l'interprétation dans le cadre soit de la doctrine, soit de la jurisprudence.

Comment pourrait-on donc demander au traducteur de rester passif devant un tel texte?

Le but que poursuit le législateur bilingue n'est pas de reproduire d'une langue à l'autre une morphosyntaxe ou une sémantique figées, mais il est avant tout de reproduire les effets de droit, c'est-à-dire les conséquences juridiques du texte de départ.

(...) Il existe des bornes à ne pas dépasser dans la liberté qu'on prend avec les formes mais ces bornes peuvent être repoussées plus loin qu'on ne le pense (Sparer, $1988: 325$ ).

Une autre particularité de la traduction juridique, à savoir le passage d'un système à un autre, est une source de difficultés supplémentaires pour le traducteur juridique, mais également une marge de liberté, une incitation à la créativité.

En effet, passer d'un système juridique à un autre signifie souvent pour le traducteur qu'il doit chercher, ou créer, des équivalences fonctionnelles, contextuelles et non pas statistiques et répétitives. Qu'il doit prendre en considération tous les aspects sociolinguistiques de son texte. Évidemment, avec le rapprochement accéléré des systèmes juridiques, avec la convergence grandissante des cultures, cette marge diminue (comme les difficultés de traduction d'ailleurs), mais elle subsiste. 
Le traducteur juridique doit prendre grand soin de son outil de travail, à savoir la langue juridique. On sait maintenant que la langue juridique est une langue de spécialité (LSP) caractérisée par une archaïcité lexicale et des particularités stylistiques, comme la prépondérance des constructions passives et réflexives et des phrases nominales et participiales. Tout cela tend à «donner une impression d'objectivité par un détachement impersonnel, artificiel, entre l'auteur du message et le récepteur» (Didier, 1987: 9). Même s'il faut considérer avec prudence la valeur universelle de ces constatations (voir par exemple les observations d'Émery (1989) concernant la langue juridique arabe), il faut avouer qu'elles constituent un obstacle à la créativité du traducteur, un facteur favorisant la standardisation de son travail. Mais si les appels à la simplification et à la modernisation de la langue juridique se multiplient dans tous les pays, y compris les milieux juridiques, si des structures sont créées à cet effet, pourquoi le traducteur resterait-il inactif? Ne pourrait-il pas devenir un des acteurs principaux de la poursuite de cet objectif?

Le postulat suprême du texte juridique est la clarté et la précision. L'application d'un des principes fondamentaux du droit, à savoir la sécurité juridique, en dépend. Et pourtant, ces textes éminemment clairs et précis (et je parle ici des textes juridiques originaux) sont la source d'une pléthore d'interprétations contradictoires. Ce n'est pas donc la servilité du traducteur à l'original qui va sauvegarder la précision et la clarté mais, au contraire, son effort rédactionnel propre qui vise les mêmes objectifs.

Ne croyons pas non plus que l'ambiguîté et l'imprécision sont bannies du royaume de la langue juridique. Au contraire, parfois la langue juridique n'est pas seulement... imprécise et ambiguë mais elle doit l'être pour favoriser une interprétation dynamique et l'adaptation à des situations qui évoluent (Gilbertson, 1988: 110). Le traducteur juridique est souvent confronté à une expérience étonnante lorsqu'il s'adresse à l'auteur d'un passage ambigu pour obtenir des éclaircissements; il reçoit souvent la réponse: «Non! Je vous en prie, surtout n'essayez pas de clarifier; il s'agit d'une ambiguîté voulue!»

Un dernier facteur intervenant dans le conflit entre standardisation et créativité dans le cadre de la traduction juridique, facteur récent mais de taille, est l'informatisation. L'informatisation du processus traductif, qui prend surtout la forme de banques de données terminologiques et de la traduction assistée par ordinateur, exige une univocité plus conséquente de la langue juridique et pousse au transcodage.

Il faut éviter à tout prix les synonymes et les périphrases qui constituent souvent un effort d'enrichissement de l'énonciation juridique. Il faut se méfier des figures rhétoriques et littéraires. Leur utilisation alourdit les programmes informatiques et rend plus difficile le travail de recherche documentaire. Le traducteur est donc... automatiquement découragé par la machine d'y recourir. Si on ajoute à cela la richesse morphologique et syntaxique de certaines langues, qui provoque aussi l'antipathie de l'ordinateur, on voit très clairement pourquoi l'informatisation de la traduction peut constituer un obstacle au travail créatif du traducteur juridique.

Pour prendre un exemple, en langue grecque, le concept de maladie peut s'exprimer en... 28 formes, si on tient compte des synonymes existant dans les deux variantes du grec moderne (la katharevoussa, la langue puriste, qui fut jusqu'en 1976 la langue officielle de l'État et la démotique, qui était la langue parlée et celle de la littérature et qui est devenue langue officielle la même année) et des différentes variations morphologiques (Passias, 1987: 141).

Par conséquent, il faut ici faire un choix: le traducteur s'efforcera ou non de faciliter le travail des informaticiens et de l'ordinateur. En tout état de cause, «le traducteur, placé devant un terme ou une expression technique, devra-t-il toujours les rendre comme le ferait la machine à traduire programmée pour sortir un équivalent au terme de la langue de départ, un mot par un mot, à la façon d'un lexique bilingue offrant un équivalent 
français pour le substituer à un terme donné en anglais (ou à quelque langue que ce soit)?» (Gémar, $1988: 313$ ).

Nous sommes convaincu que l'attitude du traducteur juridique à l'égard de son texte doit être celle d'un rédacteur créatif et non pas celle d'un ordinateur.

Pour conclure nos réflexions, nous emprunterons l'exemple proposé par l'auteur déjà cité :

Adjudication implies the application of law to individual cases brought, through one means or another, before the bar of justice.

1. Traduire selon le principe du «transcodage» (...) donnerait à peu près ceci :

Un jugement implique l'application de la loi aux causes individuelles portées, d'une manière ou d'une autre, devant la barre de justice.

2. Traduire en interprétant le texte dans l'esprit de «l'équivalence fonctionnelle» (ou de la «transparence») (...) pourrait donner, entre autres, la phrase suivante :

Rendre un jugement consiste à dire le droit dans un litige soumis à un tribunal.

(Ibid., pp. 313-314.)

Cet exemple, et l'approche suivie pour y parvenir, démontrent que le traducteur juridique, conscient des contraintes et des libertés de son travail, peut adopter une attitude rigoureuse et créative; à la question «suivre la norme ou créer?», il peut répondre, sans rechercher le paradoxe: «Créer en respectant la norme.»

\section{BIBLIOGRAPHIE}

BÉLAIR, N. (1987): «Législation bilingue - La production de la seconde version», L'Actualité terminologique, $20-6$, pp. 6-8.

DIDIER, E (1987) : «Le langage du droit et la nouvelle traduction juridique», L'Actualité terminologique, 20-5, pp. 7-11.

DUCHESNE, A. (1987): «La démarche terminologique appliquée à la common law», L'Actualité terminologique, 20-6, pp. 11-12.

ÉMERY, P.G. (1989) : «Legal Arabic Texts : Implication for Translation», Babel, 35-1, pp. 1-11.

GÉMAR, J.-C. (1988) : «La traduction juridique : art ou technique d'interprétation ?», META, 33-2, pp. 305-319.

GLBERTSON, G. (1988): «Ambiguity and Vagueness in International Law: Some German and English Examples», Lebende Sprachen, 3, pp. 110-112 et 4, pp. 162-166.

FERLAND, C. (1987): «Les bases méthodologiques de l'opération de normalisation», L'Actualité terminologique, 20-6, pp. 13-15.

KOUTSIVITIS, V. G. (1989) : «La traduction juridique, étude d'un cas : la traduction des textes législatifs des Communautés européennes, et en particulier à partir du français vers le grec», Terminologie et Traduction, 1, pp. 31-46 (résumé de thèse de doctorat).

PASSIAS, A. J. (1987): «Problèmes liés à l'élaboration d'instruments linguistiques du projet informatique INNOMOS: synonymie et polysémie dans le langage juridique hellénique», Informatique et Droit, Thessalonique, pp. 132-151.

SPARER, M. (1988): «L'enseignement de la traduction juridique : une formation technique et universitaire», META, 33-2, pp. 320-328.

WESTON, M. : «The Role of Translation at the European Court of Human Rights», Protection Human Rights : The European Dimension, Cologne, Carl Heymanns, pp. 679-689. 